\title{
Development and Evaluation of a (SEDDS) Self- Emulsifying Drug Delivery System for Darifenacin Hydrobromide
}

\author{
Nagaraja SreeHarsha1, Arshia Shariff',2, Yogesh A Shendkar², Bandar E. Al-Dhubiab1, \\ Girish Meravanige ${ }^{3}$ \\ 1Department of Pharmaceutical Sciences, College of Clinical Pharmacy, King Faisal University, Al-Ahsa, SAUDI ARABIA. \\ ${ }^{2}$ Department of Pharmaceutics, Alard College of Pharmacy, Savitribai Phule Pune University, Pune, Maharashtra, INDIA. \\ ${ }^{3}$ Department of Biomedical Sciences, College of Medicine, King Faisal University, Al-Ahsa, SAUDI ARABIA.
}

\begin{abstract}
Background: The formulation and development of new chemical entities has the major challenge of low solubility. A fraction of newly manufactured drugs (40\%) have poor hydrophilicity. As a result, the delivery of these drugs bioavailability, thus limiting the rate of absorption of hydrophobic drugs. Method: Self-Emulsifying Drug Delivery (SEDDS) system with the poorly hydrophilic drug, darifenacin was developed. We conducted solubility studies to obtain the materials that allowed for the maximum solubility of darifenacin. Results: The highest solubility was found to be labrafil 1944 CS (Surfactant) polyethylene glycol 400 (Co-surfactant) and peanut oil. Emulsion regions were evaluated in constructed ternary phase diagrams. Thermodynamic stability and phase separation studies were conducted to investigate the degree of phase separation of the various formulations. The average globule size of SEDDS was witnessed to be less than 200 $\mathrm{nm}$ for in our optimized formulations and exhibiting negative zeta potential. When we compared the dissolution of emulsion formulations to pure darifenacin and the results showed that the rate of dissolution in the developed formulations with darifenacin was increased as compared to pure drug. Conclusion: Thus, SEDDS may provide a viable alternative for existing formulations of darifenacin on the market.
\end{abstract}

Key words: Self-Emulsifying Drug Delivery (SEDDS), Darifenacin, Peanut Oil, Labrafil M 1944, Polyethylene Glycol 400, Ternary Phase Diagrams.

\section{INTRODUCTION}

Overactive Bladder Syndrome (OAB), as defined by the International Continence Society (ICS) is experiencing "urgency", with or without urgency urinary incontinence and is often asosicated with urinary frequency and nocturia. ${ }^{1}$ Pathophysiologically, $\mathrm{OAB}$ is caused by an unintentional contraction of the detrusor muscle in the bladder, which is controlled by activation of the muscarinic receptors on the surface of the detrussor muscle of the bladder. ${ }^{2,3}$ There are plenty of theories explaining the etiology of the syndrome but it is generally agreed that the factors involved are either myogenic, neurogenic and/or integrative (trigger-mediated). ${ }^{4} \mathrm{OAB}$ may have a deleterious effect on quality of life since it considerably affects the occupational, physical and sexual activities of the patients. Thus, it can lead to impaired work productivity, sleep disturbances, social isolation and depression ${ }^{5} \mathrm{OAB}$ with urge incontinence is also a major reason for hospital admissions among the elderly. ${ }^{1}$

$\mathrm{OAB}$ is equally prevalent in men and women and mainly affects the elderly. The incidence significantly increases with age, as some $40 \%$ of women who are postmenopausal are affected. ${ }^{6}$ The rapidly changing social, economic and demographics in Saudi
Submission Date: 01-03-2019; Revision Date: 20-03-2019; Accepted Date: 01-04-2019.

DOI: 10.5530/ijper.53.2s.67 Correspondence: Dr. Sree Harsha Nagaraja, Department of Pharmaceutical Sciences, College of Clinical Pharmacy, King Faisal University, Al-Ahsa, SAUDI ARABIA.

Phone: +96-653-548-5322

E-mail: sharsha@kfu.edu.sa

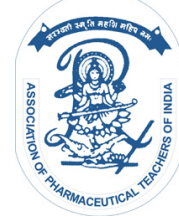

www.ijper.org 
Arabia indicate longer life spans and declined fertility rates. The United Nations foresee that the population of Saudi Arabians above 65 years old will increase dramatically and the elderly will make up $18.4 \%$ of the total population in 2050.7 Therefore, our present work is aimed at significantly benefiting the elderly population of Saudi Arabia, commonly affected by this debilitating disease.

The most common cause of $\mathrm{OAB}$ remains over activity of the detrusor muscle of the bladder. The activity of the urinary bladder is predominantly controlled by M2 and M3 muscarinic receptors, with M3 receptors being mainly responsible for detrusor contractility and consequently OAB. ${ }^{8}$ Available anticholinergic agents do exist but they lack selectivity for the receptors on the detrussor muscle on the bladder and cause systemic anticholinergic peripheral side effects including dry mouth, blurred vision and constipation. ${ }^{9}$ As such, new anticholinergic drugs such as Darifenacin and Solifenacin that are more selective for the muscarinic M3 bladder receptor are preferred for oral therapy. ${ }^{10}$ These active agents have a high affinity for M3 receptors as compared to other muscarinic receptors and have been proven to be safe for $\mathrm{OAB}$ therapy. However, they are very lipophilic and suffer from low dissolution rates and consequently poor absorption and bioavailability. ${ }^{2}$ One of the common ways to increase the rate of oral absorption of exceptionally lipophilic drugs is to incorporate them in Self-Emulsifying Drug Delivery Systems (SEDDS). SEDDS are isotropic mixtures of oils, solvents, surfactants and co-solvents/surfactants that can be given in hard or soft gelatin capsules. ${ }^{11}$ They are quickly distributed in the fluids of the gastrointestinal tract, yielding microemulsions or nanoemulsions that contain the solubilized drug. Due to its small size, the drug can be absorbed through the lymphatic system, thereby avoiding first-pass metabolism. ${ }^{12}$ SEDDS exhibit remarkable physical stability in contrast to most dispersed forms that are unstable and sensitive. Furthermore, the manufacturing process of SEDDS is simple. However, Their performance is highly dependent on the design of the formulation, as limited excipient combinations can result in effective SEDDS. ${ }^{11}$

In this study, we focused on the formulation of SEDDS containing highly lipophilic M3 muscarinic antagonists. Various excipients were examined during the pre-formulation studies to examine the suitability of drugs, oils, surfactants and co-solvents. We proceeded with the formulation of SEDDS which were then evaluated in vitro for size distribution, droplet size, zeta potential, the effect of digestion, emulsification times, robustness to dilution and stability testing. Moreover, we examined the drug content and the efficiency of drug loading and tested the in-vitro release profile of the drug.

The objective of our study was thus to formulate selective M3 muscarinic antagonists into a SEDDS forumation that would increase the bioavailability and favour drug distribution of Darifenacin through increased interfacial area. We believe that this formulation will also concurrently reduce the systemic side effects of nonselective anti-muscarinic drugs as well. ${ }^{13}$

\section{MATERIALS AND METHODS}

\section{Materials}

We received Darifenacin as a gift from Emcure Pharmaceuticals, Pune, India. From Gattefosse Corp. (SaintPriest Cedex, France), we obtained free samples of Capryol-90, Labrasol, Lauroglycol 90, Labrafil ${ }^{\circledR}$ M 1944 CS and Labrafac ${ }^{\text {TM }}$ lipophile WL-1349. All chemicals and solvents were analytical grade reagents. Finally, we purchased peanut oil from the local market.

\section{Preformulation Study}

\section{Solubility Determination}

To determine the solubility of darifenacin in various oil, surfactants and co-surfactants, we dissolved the drug in $2 \mathrm{ml}$ of vehicle. The samples were vortexed and incubated at room temperature for $75 \mathrm{hrs}$ and to ensure that we achieved maximal solubility. We then centrifuged the samples at a speed of $3000 \mathrm{rpm}$ for 15 mins. Using UVspectrophometer, we determined the concentration of the drug in each vehicle. ${ }^{14}$

\section{Preparation of Pseudo-Ternary Phase Diagrams}

To construct pseudo-ternary phase diagrams, we used the water titration method with different weight various ratios of oil, surfactant: co-surfactant. We used various surfactant: cosurfactant ratios $(1: 1,1: 2,1: 3,1: 4,2: 1,3: 1$, 4:1) named as $\mathrm{S}_{\text {mix }}$. Furthermore, various oil: $\mathrm{S}_{\text {mix }}$ ratios were prepared (1:1, 1:2, 1:3, 1:4, 1:5, 1:6, 1:7, 1:8, 1:9). We constructed the phase diagrams of oil: $\mathrm{S}_{\text {mix }}$, water was used to titrate the mixture in a dropwise manner. ${ }^{15}$ Once we identified the emulsion region, we carried out the construction using chemix school 7 software and selected emulsion formations at desired ratios.

\section{Formulation of SEDDS Containing Darifenacin}

We used Labrafil M 1944 CS and PEG 400 to prepare formulations of surfactant and cosurfactant with Smix ratio 2:1 (Table 1). The final mass of the formulation was maintained at $1 \mathrm{~g}$, while the amount of darifenacin was maintained at $7.5 \mathrm{mg}$ in all rounds of SEDDS. Darifenacin and peanut oil were gently stirred and mixed 
with vortex. Calculated amounts of surfactant and cosurfactant quantities placed in a vial and gently mixed. This mixture was heated to $40^{\circ} \mathrm{C}$ at which we observed complete dissolution of darifenacin. ${ }^{16}$ Aerosil 200 was used as the carrier for the liquid SEDDS. ${ }^{17}$

\section{Evaluation of SEDDS}

\section{Phase Separation study}

We diluted each round of SEDDS in $200 \mathrm{~mL}$ of water (distilled) at $37 \circ \mathrm{C}$ and examined the physical appearance of the solution. The dilution was then mixed by vortex for 1 mins and stored for $24 \mathrm{hrs}$. We visually observed the phase separation. ${ }^{18}$ For subsequent studies, we used mixtures that had exhibited minimal to no phase separation.

\section{Thermodynamic Stability Studies}

To evaluate the stability of SEDDS, we used a number of freeze thaw cycles. Formulations underwent 3-4 freezing/thawing cycles, with a cold temperature of $4^{\circ} \mathrm{C}$ for $24 \mathrm{hr}$ followed and a warmer temperature of $40^{\circ} \mathrm{C}$ for $24 \mathrm{hr}$. We centrifuged all preparations and waited to observe if phase separation occurred. ${ }^{15}$

\section{Self-Emulsificatying Time}

We examined the SEDDS formulations for any indication of properties of self-emulsification. Visual assessment was carried out based on apparent stability and clarity of the resultant emulsion. SEDDS, the pre-concentrate, was added to $250 \mathrm{ml}$ of distilled water, dropwise and magnetically perturbed at a gentle rate of 125 $\mathrm{rpm}$. All solutions were visually inspected and subsequently graded. ${ }^{16,18}$

\section{Robustness to Dilution}

To detect if there would be precipitation in reconstituted SEDDS with increase of dilution extent from 10 times with water. We diluted the SEEDS to 10 and did this for 100 rounds using $25 \mathrm{ml}$ of water and stored it for 12 hrs. ${ }^{19}$ We then observed whether the dilution emulsions demonstrated any phase separation or precipitation.

\section{Effect of $\mathrm{pH}$ of Dilution Media}

Three different dissolution medium, viz. water and phosphate buffers with $\mathrm{pH}$ value of 1.2, 4.5 and 6.8 we diluted the SEDDS to 10 parts and then stored the dilutions for $12 \mathrm{hrs}$ and observed for any clouldiness, separations or precipitation. ${ }^{19}$

\section{Analysis of Particle Size}

The particle size of reconstituted formulation of SEDDS was mixed gently and then analysed using the Malvern Zetasizer Nano ZS instrument (Malvern Instruments, Malvern, UK). ${ }^{15,19,20}$

\section{Determination of Zeta Potential}

To determine the zeta Potential of the suspension, we diluted 1:10 (SEDDS:water) in a beaker, mixing with a magnetic stirrer at a constant speed. Using a Malvern Zetasizer (Nano ZS 90, Worcesterhire UK), we assessed both the electrophoretic mobility and zeta-potential of the formulated emulsion. ${ }^{19}$

\section{Dissolution Studies Carried out in vitro}

Using the USP apparatus II, dissolution studies were carried out. The " 00 " capsule size were taken and filled with SEDDS of darifenacin and examined the profiles of in vitro release of both SEDDS of darifenacin and pure darifenacin in powdered form. Each capsule were placed in five vessels. The pure drug was placed in a vessel with the dissolution apparatus that had $900 \mathrm{ml}$ of water and the temperature was constant at $37 \pm 0.5^{\circ} \mathrm{C}$. At predetermined time intervals $(5,10,20,30,40,60$ and $70 \mathrm{~min})$ the sample were withdrawn and diluted

\begin{tabular}{|c|c|c|c|c|c|c|c|c|c|}
\hline $\begin{array}{l}\text { Preparation } \\
\text { codel } \\
\text { components } \\
\text { (mg) }\end{array}$ & $F-1$ & F-2 & $F-3$ & $F-4$ & $F-5$ & F-6 & F-7 & $F-8$ & F-9 \\
\hline Darifenacin & 7.5 & 7.5 & 7.5 & 7.5 & 7.5 & 7.5 & 7.5 & 7.5 & 7.5 \\
\hline Smix ratio & \multicolumn{9}{|c|}{$2: 1$} \\
\hline Oil: Smix & $1: 1$ & $1: 2$ & $1: 3$ & $1: 4$ & $1: 5$ & $1: 6$ & $1: 7$ & $1: 8$ & $1: 9$ \\
\hline Peanut oil & 496.25 & 330.83 & 248.12 & 198.5 & 165.41 & 141.75 & 124.06 & 110.27 & 99.25 \\
\hline $\begin{array}{c}\text { Labrafil M } \\
1944 \text { CS }\end{array}$ & 330.83 & 441.10 & 496.24 & 529.33 & 551.38 & 567.14 & 578.95 & 588.14 & 595.5 \\
\hline PEG 400 & 165.41 & 220.55 & 248.12 & 264.66 & 275.69 & 283.57 & 289.47 & 294.07 & 297.75 \\
\hline Total & 1000 & 1000 & 1000 & 1000 & 1000 & 1000 & 1000 & 1000 & 1000 \\
\hline
\end{tabular}


with distilled water and absorbance were measured at $287 \mathrm{~nm}$ wavelength. ${ }^{21}$

\section{Drug Excipient Compatibility Studies}

Interaction studies were carried out using FTIR spectrophotometer (Shimadzu) for both Darifenacin API and Darifenacin formulations at range of $400-4000 \mathrm{~cm}^{-1} .{ }^{14}$

\section{RESULTS AND DISCUSSIONS Preformulation Study \\ Solubility Studies}

Formulation of SEDDS were prepared to increase the solubility / oral bioavailability of the drug. As such, it was necessary for each component to have a high capacity for solubilisation to measure the maximal drug loading and decrease the final dosing volume. In Table 2 and Figure 1, we present the solubility of darifenacin in different vehicles. Here we used peanut oil as the oil phase, while Labrafil ${ }^{\circledR} 1944$ CS and Polyethylene Glycol 400 were used as the surfactant and cosurfactant respectively. Both provided a high degree of solubility. Throughout our studies, it was paramount to select components that are highly soluble to ensure the successful formulation.

\section{Construction of Phase Diagram}

To determine the optimal concentration of surfactant, co-surfactant and oil, we plotted phase diagrams. We prepared several formulations using peanut oil. We also used seven different Smix ratios of 1:1, 1:2, 1:3, 1:4, 2:1, 3:1 and 4:1 for Labrafil ${ }^{\circledR} 1944$ CS and PEG 400. Each mixture was titrated with water to obtain regions of nanoemulsion. We observed such regions visually and made various nanoemulsion gradings as follows: milky gel with medium flow: emulgel $(\mathrm{M})$, oil/water (N), cloudy with good flow: emulsion (E) transparent gel with medium flow: nanoemulsion gel $(G)$, We then constructed phase diagrams as shown in Figure 2 (a-g) and also compared the regions of nanoemulsion that had various ratios of surfactant: cosurfactant (Smix ratios). In a ternary phase diagram, a large region of nanemulsion is often associated with a greater efficiency of self-emulsification. In the figure, the 2:1 Smix ratio demonstrated the largest region of nanoemulsification. As such, we selected this Smix ratio of 2:1 for further studies.

\section{Characterization of SEDDS Visual Test}

A series of SEDDS with distilled water were prepared and observed the phase separation. We found that formulations SF1, SF2, SF3, SF4, SF5 demonstrated no evidence of phase separation (Table 3). As we needed stable formulations for further studies, we only selected those that did not exhibit phase separation.

\section{Thermodynamic Stability Studies}

A series of thermodynamic stability studies and stress tests were executed. We found that the formulations: SF1, SF2, SF3 and SF4, were thermodynamically stable (Table 4). Furthermore, during the stress test, these formulations also did not exhibit any evidence of phase separation.

\section{Self-Emulsification Assessment}

The efficacy of the self-emulsification study was also assessed. Our studies demonstrated that formulations of SF1, SF2, SF3 and SF4 formed microemulsions within a minute. These microemulsions were both rapid and clear. However, while the formulation SF5 formed a microemulsion rapidly, it was less clear as compared to formulations SF1-SF4 (Table 5).

\section{Robustness to Dilution}

To obtain a stable microemulsion of the SEDDS formulation, it is important to obtain the right blend of emulsifier. We diluted the SEDDS formulations of SF1, SF2, SF3 and F\$ to 10, 100 times with $\mathrm{H}_{2} \mathrm{O}$. We observed a lack of phase separation or drug precipitation from any of the diluted SEDDS. As such, all of our formulations demonstrated a robustness to dilution using aqueous solution.

\section{Effect of $\mathrm{pH}$}

The SEDDS formulations (SF1, SF2, SF3 and SF4) were diluted using phosphate buffer with $\mathrm{pH}$ values of

\begin{tabular}{|c|c|}
\hline Table 2: Darifenacin solubility studies in Surfactants, \\
co-surfactants and Various Oils. \\
\hline Vehicle & $\begin{array}{c}\text { Darifenacin Solubility at } \mathbf{2 5}^{\circ} \mathbf{C} \\
\text { ( } \mathbf{m g} / \mathbf{m l} \text { ) }\end{array}$ \\
\hline Surfactants & \\
Capryol 90 & 1.1934 \\
Lauroglycol & 1.2096 \\
Labrasol & 1.3852 \\
Labrafil 1944 CS & 3.8401 \\
Cosurfactants & 1.3689 \\
Ethylene glycol & 1.5534 \\
Ethanol & 2.1057 \\
PEG 400 & 1.7475 \\
Propylene glycol & \\
Oils & 2.3538 \\
Cotton seed oil & 0.9196 \\
Castor oil & 0.3606 \\
Labrafac lipophile WL 1349 & 4.8467 \\
Peanut oil & 0.9715 \\
Soybean oil & 0.9074 \\
Sunflower oil &
\end{tabular}




Table 3: Visual Observation, Phase Separation of Emulsion.
\begin{tabular}{|c|c|c|c|c|c|c|c|c|c|}
\hline Formulation code & $\begin{array}{c}\text { SF-1 } \\
(1: 9)\end{array}$ & $\begin{array}{c}\text { SF-2 } \\
(1: 8)\end{array}$ & $\begin{array}{c}\text { SF-3 } \\
(1: 7)\end{array}$ & $\begin{array}{c}\text { SF-4 } \\
(1: 6)\end{array}$ & $\begin{array}{c}\text { SF-5 } \\
(1: 5)\end{array}$ & $\begin{array}{c}\text { SF-6 } \\
(1: 4)\end{array}$ & $\begin{array}{c}\text { SF-7 } \\
(1: 3)\end{array}$ & $\begin{array}{c}\text { SF-8 } \\
(1: 2)\end{array}$ & $\begin{array}{c}\text { SF-9 } \\
(1: 1)\end{array}$ \\
\hline Phase separation & $\infty$ & $\infty$ & $\infty$ & $\infty$ & $\infty$ & $\beta$ & $B$ & $B$ & $\beta$ \\
\hline
\end{tabular}

$(\infty:$ No phase separation; $\beta$ Phase separation).

\begin{tabular}{|c|c|c|}
\hline \multicolumn{2}{|c|}{ Table 4: Stability Studies at $-4^{\circ} \mathrm{C}$ and $40^{\circ} \mathrm{C}}$. \\
\hline Formulation & Storage at $-4^{\circ} \mathrm{C}$ & Storage at $40^{\circ} \mathrm{C}$ \\
\hline SF-1 &,--- &,--- \\
\hline SF-2 &,--- &,--- \\
\hline SF-3 &,--- &,--- \\
\hline SF-4 &,--- &,--- \\
\hline SF-5 & ++ & ++ \\
\hline
\end{tabular}

(-- No precipitation, ++ precipitation, + Phase separation, - No phase separation).

\begin{tabular}{|c|c|c|c|c|c|}
\hline \multicolumn{7}{|c|}{ Table 5: Self-Emulsification Assessment. } \\
\hline Formulations & SF-1 & SF-2 & SF-3 & SF-4 & SF-5 \\
\hline Clarity & $\begin{array}{c}\text { Clear and } \\
\text { monophasic }\end{array}$ & $\begin{array}{c}\text { Clear and } \\
\text { monophasic }\end{array}$ & $\begin{array}{c}\text { Clear and } \\
\text { monophasic }\end{array}$ & $\begin{array}{c}\text { Clear and } \\
\text { monophasic }\end{array}$ & Turbid \\
\hline
\end{tabular}

\begin{tabular}{|c|c|c|c|c|c|c|}
\hline \multicolumn{3}{|c|}{ Formulation code } & \multirow{2}{*}{$\begin{array}{c}\text { SF1 } \\
(1: 9) \\
-\end{array}$} & \multirow{2}{*}{$\begin{array}{c}\text { SF2 } \\
(1: 8) \\
-\end{array}$} & \multirow{2}{*}{$\begin{array}{c}\text { SF3 } \\
(1: 7) \\
-\end{array}$} & \multirow{2}{*}{$\begin{array}{c}\text { SF4 } \\
(1: 6) \\
-\end{array}$} \\
\hline \multirow{6}{*}{$\begin{array}{c}\text { Drug } \\
\text { Precipitation }\end{array}$} & \multirow[t]{2}{*}{ PH 1.2} & 10 Times & & & & \\
\hline & & 100 Times & - & - & - & - \\
\hline & \multirow[t]{2}{*}{ PH 4.5} & 10 Times & - & - & - & - \\
\hline & & 100 Times & - & - & - & - \\
\hline & \multirow[t]{2}{*}{ PH 6.8} & 10 Times & - & - & - & - \\
\hline & & 100 Times & - & - & - & - \\
\hline
\end{tabular}

(- indicates absence of drug precipitation).

1.2, 4.5 and 6.8. We diluted them to 10,100 times with this buffer. In Table 6, we demonstrated that none of the diluted SEDDS demonstrated any phase separation or drug precipitation on storage. As such, our data demonstrates that the diluted media were stable with varying dilutions and at varying $\mathrm{pH}$ values that represent the $\mathrm{pH}$ range of the GI.

\section{Droplet Size Analysis}

It is thought that droplet size is related to the concentration of surfactants in the experiment. As such, an increase in the concentration of surfactant could be observed to lead to small droplet sizes on average. This phenomenon is due to the surfactants being confined at the oil-water interface. However, the average size of the droplet may increase with an increase in the concentration of the surfactants. This is thought to be due to interfacial disruption due to increased water penetration into the droplets of oil. This could be due to the increase in the concentration of surfactant and subsequent oil being ejected into the aqueous phase. The determination of the size of participles that followed self-micro emulsification is an important factor for evaluating the system of self-microemulsion. This is because the size of the droplet is assumed to affect the rate of drug absorption. As such, a droplet of smaller size has larger surface area of interface for drug absorption. ${ }^{4}$

In Table 7 and Figures 3-6, we summarized the average size of droplets and PDI for all the SEDDS. We measured polydispersity as the ratio of the standard devia- 


\begin{tabular}{|c|c|c|c|c|}
\hline \multicolumn{5}{|c|}{ Table 7: Emulsion Droplet Size of SEDDS. } \\
\hline Formulation code & $\begin{array}{c}\text { SF-1 } \\
(\mathbf{1 : 9 )}\end{array}$ & $\begin{array}{c}\text { SF-2 } \\
\mathbf{( 1 : 8 )}\end{array}$ & $\begin{array}{c}\text { SF-3 } \\
\mathbf{( 1 : 7 )}\end{array}$ & $\begin{array}{c}\text { SF-4 } \\
\mathbf{( 1 : 6 )}\end{array}$ \\
\hline $\begin{array}{c}\text { Emulsion globule size } \\
(\mathrm{nm})\end{array}$ & 129.3 & 704.2 & 435.7 & 149.5 \\
\hline PDI & 0.276 & 1.000 & 1.000 & 0.234 \\
\hline
\end{tabular}

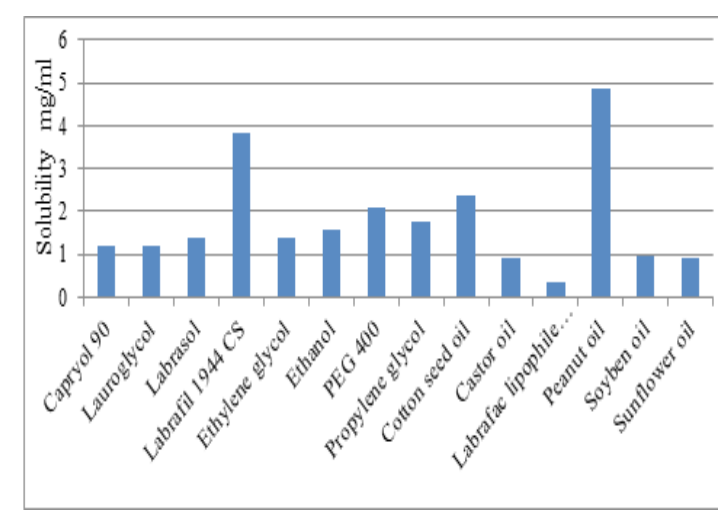

Figure 1: Drug Solubility in Different Oils, Cosurfactants and Surfactants.

tion to the average size of droplet. This analysis helps to find out the consistency of the droplet size that is present within the formulation. There is a negative correlation, as the higher value of polydispersity mean a lower degree of uniformity in the formulation's droplet size. Examining Smix formulation ratios of 1:9 and 1:6 demonstrated that the lowest emulsion droplet size and PDI. As such, we employed the use of the SF1 and SF4 formulations in our in-vitro release studies.

\section{Zeta Potential Determination}

In Table 8 and Figures $6-10$, we demonstrate that the SEDDS report negatives values in the zeta potential. In fact, the surfactant (Labrafil M 1944 CS) and cosurfactant (PEG 400) employed in these experiments were all non-ionic and were non-contributory to the total overall charge in the microemulsion particle. Our results show that the Zeta potential values in the $-30 \mathrm{mv}$ to $+30 \mathrm{mv}$ range are actually stable.

\section{In vitro Dissolution Studies}

Figure 8-15, we demonstrate the results of the profiles of in vitro dissolution of the various formulations. Our results demonstrate an improvement in SEDDS formulation in camparsion with Darifenacin pure in media.

Drug Excipient Compatibility studies

To assess for possible drug and excipient interactions, we carried out FTIR studies. We obtained infrared spectrums of both the plain drug and selected formulations.
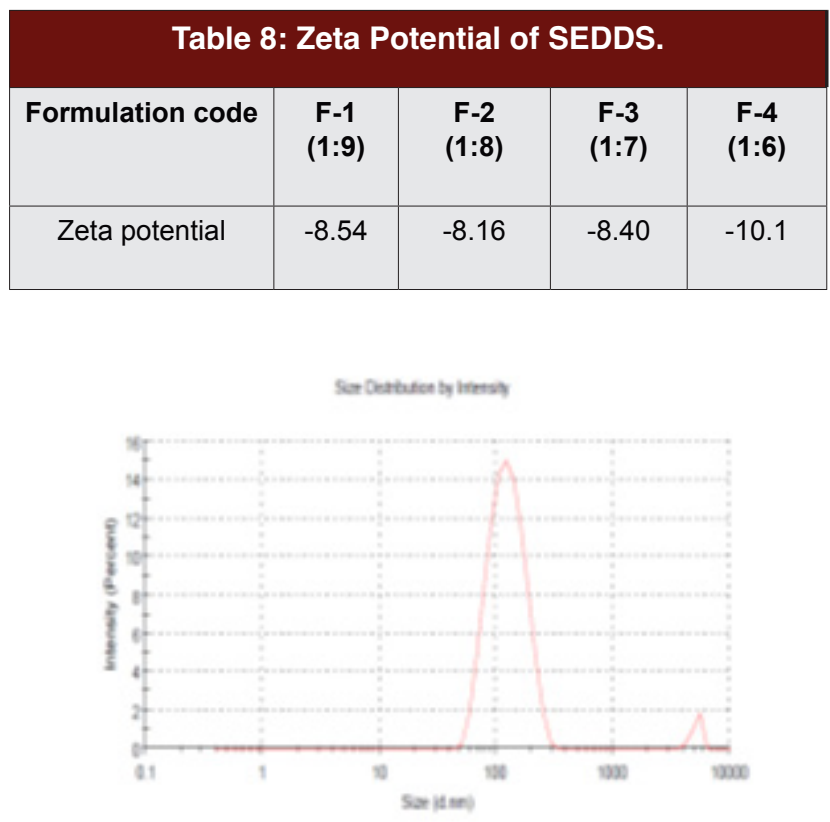

Figure 3: Size and PDI of SF1.

Our results are shown in Figures 12 and 13. Our studies showed the FTIR spectra of Darifenacin Hydrobromide and showed retained peaks with modest shifts. We

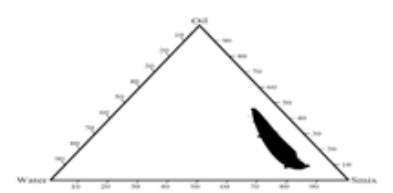

A) Ratio 1:1

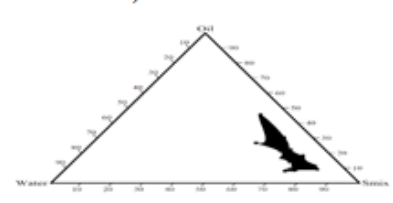

C) Ratio $1: 3$

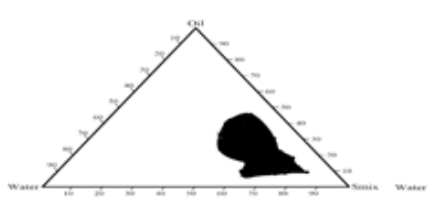

E) Ratio 2:1

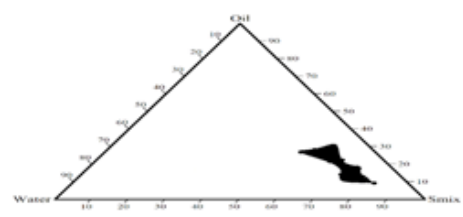

G) Ratio 4:1

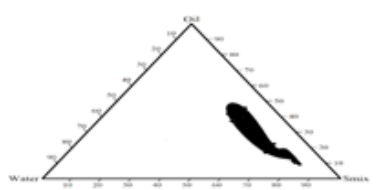

B) Ratio $1: 2$

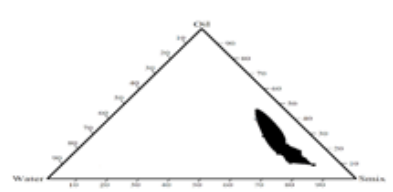

D) Ratio 1:4

F) Ratio $3: 1$

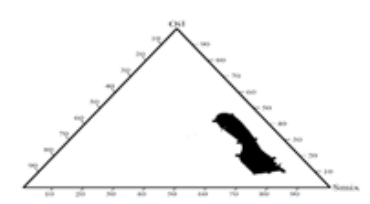

Figure 2: Phase Diagram Prepared with (A-G) Peanut Oil, Labrafil 1944 CS, PEG 400 Systems in Presence of Darifenacin Indicating Nanoemulsion Phase. 


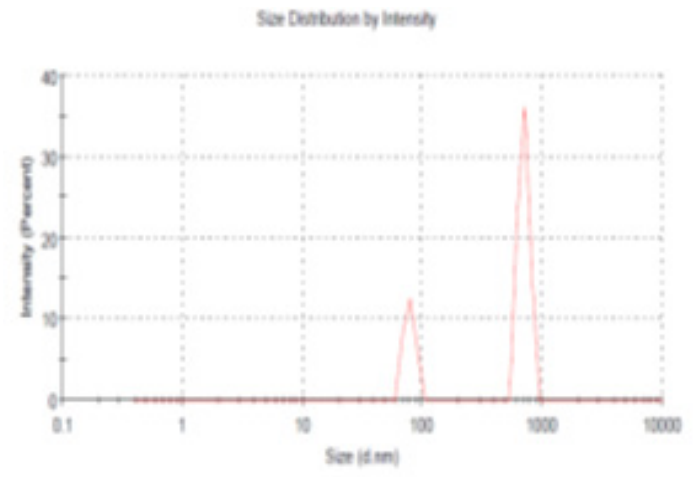

Figure 4: Size and PDI of SF2.

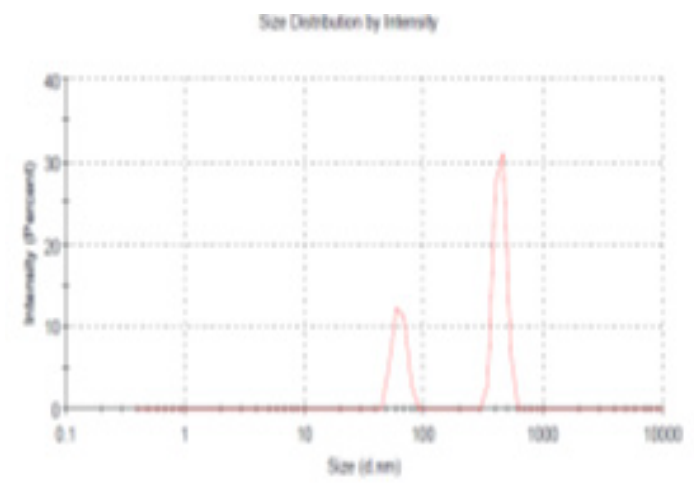

Figure 5: Size and PDI of SF3.

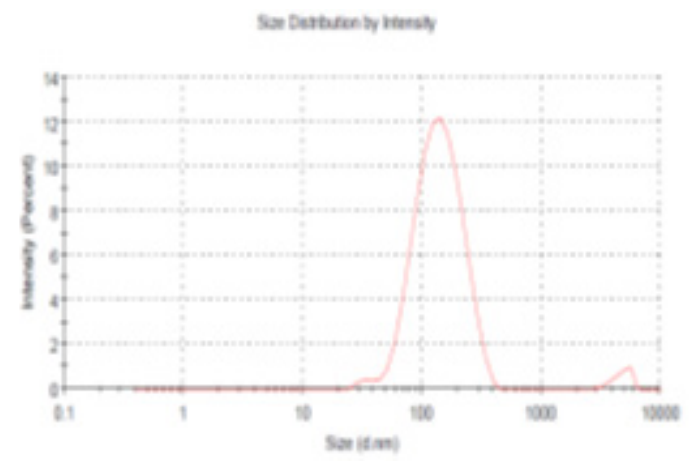

Figure 6: Size and PDI of SF4.

interpreted our results to mean that our formulation is stable and retained drug functionality capabilities.

\section{CONCLUSION}

Our preformulation studies yielded characterization results of Darifenacin that were in compliance with the certificate of analysis provided by the sample provider. Our calibration curves demonstrated that Darifenacin follows Beer-Lambert's law. Finally, the FTIR spectra

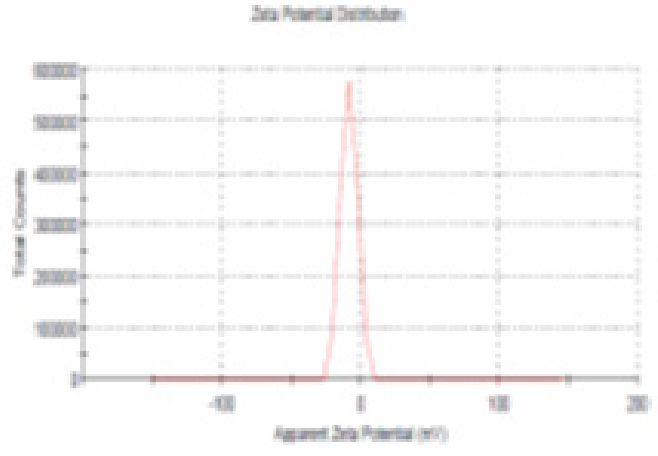

Figure 7:Zeta Potential of SF1.

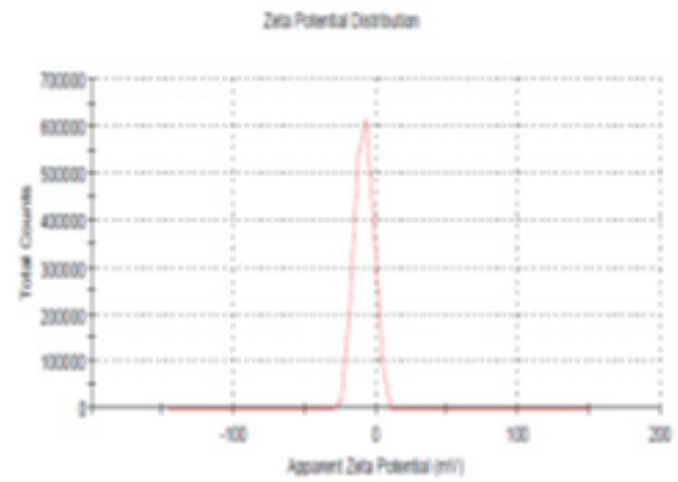

Figure 8: Zeta Potential of SF2.

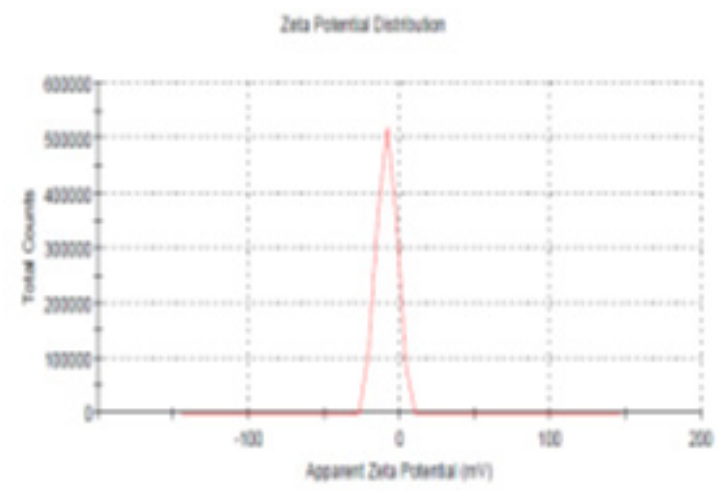

Figure 9: Zeta Potential of SF3.

demonstrated characteristic peaks. Overall, our starting drug samples were pure.

We selected surfactant, oil and co-surfactant materials and constructed ternary phase diagrams using various Smix ratios. The Smix ratios used were 1:1, 1:2, 1:3, 1:4, 2:1, 3:1 and 4:1. The analyses of the ternary phase diagram confirmed that the Smix ratio of 2.1 yeilded optimal results. As such, the surfactant to cosurfactant ratio of 2:1 was chosen to prepare the liquid SEDDS of these drugs. 


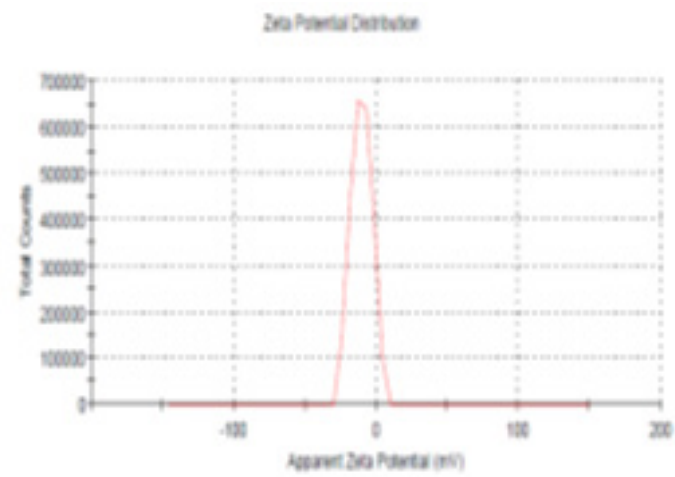

Figure 10: Zeta Potential of SF4.

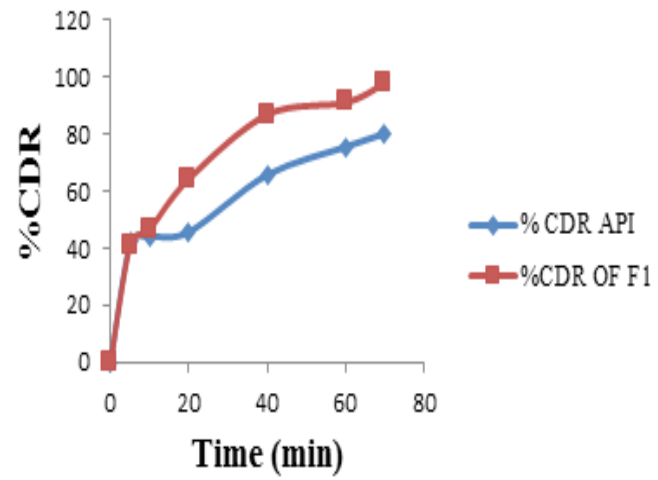

Figure 11: Profile of in vitro dissolution of Pure Darifenacin and SF1.

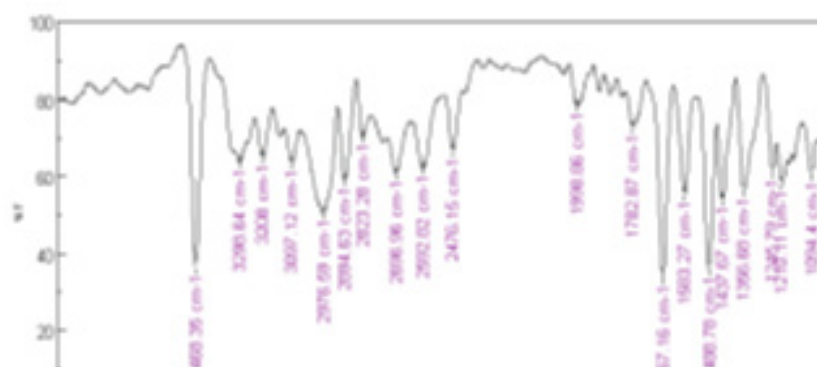

Figure 12: FTIR Spectra of Darifenacin Hydrobromide.

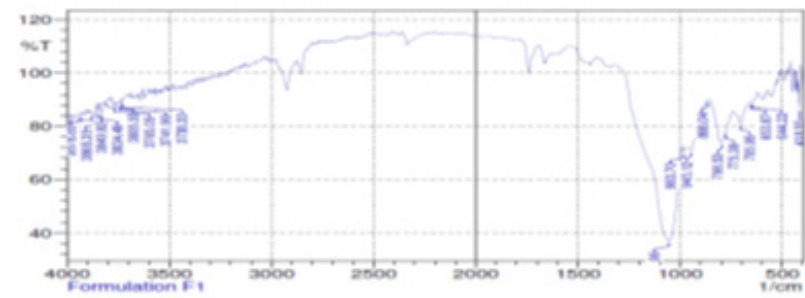

Figure 13: FTIR Spectra of SF1 SEDDS.
Additionally, a series of studies were carried out including: visual assessments, thermodynamic stability studies, robustness to dilution and assessment of the efficiency of self-emulsification. Our results demonstrated that it was only the SF1, SF2, SF3 and SF4 formulations of SEDDS that were stable.

Our studies demonstrated that the globule size of formulations were as follows: $\mathrm{SF} 1=-129.3, \mathrm{SF} 2=-704.2$, $\mathrm{SF} 3=-435.7$ and SF4 $=-149.5 \mathrm{~nm}$. We found the zeta potential of formulations to be: $\mathrm{SF} 1=-8.54, \mathrm{SF} 2=-8.16$, $\mathrm{SF} 3=-8.40$ and SF4 $=-10.1 \mathrm{mV}$. Finally, the polydispersity index of SF1 and SF4 formulations each had a value of $<1$, the uniform distribution of globules throughout the formulation. As such, we only selected the formulations SF1 and SF2 for further studies. Our studies demonstrated that the dissolution rate of SEDDS was more efficient as compared to pure Darifenacin and in compatibility studies, the SEDDS formulation of Darifenacin retained the functional group, showing retained functionality. Overall, our work demonstrates that prepared formulations SEDDS are possible, producing enhanced rates of dissolution and absorption.

\section{ACKNOWLEDGEMENT}

This study was funded by Deanship of Scientific Research, King Faisal University, Al-Ahsa, Kingdom of Saudi Arabia. (Grant Number: 130014).

\section{CONFLICT OF INTEREST}

The authors declare no conflict of interest.

\section{REFERENCES}

1. Dokmeci F, Cetinkaya SE, Seval MM, et al. Ambulatory urodynamic monitoring of women with overactive bladder syndrome during single voiding cycle. Eur J Obstet Gynecol Reprod Biol. 2017;212:126-131. 2017/03/30. DOI: 10.1016/j.ejogrb.2017.03.023.

2. Brunton S, Kuritzky L. Recent developments in the management of overactive bladder: focus on the efficacy and tolerability of once daily solifenacin succinate $5 \mathrm{mg}$. Current Medical Research and Opinion. 2005;21(1):71-80. DOI: 10.1185/030079904X20268.

3. Cipullo LM, Cosimato C, Filippelli A, et al. Pharmacological approach to overactive bladder and urge urinary incontinence in women: An overview. Eur J Obstet Gynecol Reprod Biol. 2014;174:27-34. 2014/01/15. DOI: 10.1016/j. ejogrb.2013.12.024.

4. Tyagi $\mathrm{P}$, Kashyap $\mathrm{M}$, Yoshimura $\mathrm{N}$, et al. Past, Present and Future of Chemodenervation with Botulinum Toxin in the Treatment of Overactive Bladder. J Urol. 2017;197(4):982-90. 2016/11/23. DOI: 10.1016/j. juro.2016.11.092.PMC5386789.

5. Abrams P, Kelleher CJ, Kerr LA, et al. Overactive bladder significantly affects quality of life. Am J Manag Care. 2000;6(11 Suppl):S580-90. 2001/02/24.

6. Macdiarmid SA. Maximizing the treatment of overactive bladder in the elderly. Reviews in Urology. 2008;10(1):6-13. 
7. Karlin NJ, Weil J, Felmban W. Aging in Saudi Arabia: An Exploratory Study of Contemporary Older Persons' Views About Daily Life, Health and the Experience of Aging. Gerontology and Geriatric Medicine. 2016;2:2333721415623911. DOI: 10.1177/2333721415623911.

8. Starkman JS, Dmochowski RR. Management of overactive bladder with transdermal oxybutynin. Reviews in Urology. 2006;8(3):93-103.

9. Hashim H, Abrams P. Drug treatment of overactive bladder: efficacy, cost and quality-of-life considerations. Drugs. 2004;64(15):1643-56. 2004/07/20. DOI: 10.2165/00003495-200464150-00003.

10. Haab F, Stewart L, Dwyer P. Darifenacin, an M3 selective receptor antagonist, is an effective and well-tolerated once-daily treatment for overactive bladder. Eur Urol. 2004;45(5):420-9. discussion 429. 2004/03/26. DOI: 10.1016/j. eururo.2004.01.008

11. Neslihan GR, Benita S. Self-emulsifying drug delivery systems (SEDDS) for improved oral delivery of lipophilic drugs. Biomedicine and Pharmacotherapy. 2004;58(3):173-82. DOI: https://doi.org/10.1016/j.biopha.2004.02.001.

12. Singh B, Bandopadhyay $\mathrm{S}$, Kapil $\mathrm{R}$, et al. Self-emulsifying drug delivery systems (SEDDS): formulation development, characterization and applications. Crit Rev Ther Drug Carrier Syst. 2009;26(5):427-521. 2010/02/09.

13. Pathak $A$, Jain $\mathrm{V}$, Nagariya $\mathrm{AK}$, et al. Recent advances in self emulsifying drug delivery system - A review. Drug Invention Today. 2010;2(2):123- 9.

14. Hyma $P$ and Abbulu K. Formulation and characterisation of selfmicroemulsifying drug delivery system of pioglitazone. Biomedicine and Preventive Nutrition. 2013;3:345-50. DOI: https://doi.org/10.1016/j. bionut.2013.09.005
15. Shafiq-un-Nabi S, Shakeel F, Talegaonkar S, et al. Formulation development and optimization using nanoemulsion technique: a technical note. AAPS Pharm Sci Tech. 2007;8:E12-28. DOI: 10.1208/pt0802028

16. Patel AR and Vavia PR. Preparation and in vivo evaluation of SMEDDS (selfmicroemulsifying drug delivery system) containing fenofibrate. The AAPS Journal. 2007;9:E344-E52. DOI: 10.1208/aapsj0903041

17. Balakrishnan $\mathrm{P}$, Lee $\mathrm{BJ}$, Oh $\mathrm{DH}$, et al. Enhanced oral bioavailability of dexibuprofen by a novel solid self-emulsifying drug delivery system (SEDDS). Eur J Pharm Biopharm. 2009;72:539-45. 2009/03/21. DOI: 10.1016/j. ejpb.2009.03.001

18. Khoo SM, Humberstone AJ, Porter $\mathrm{CJH}$, et al. Formulation design and bioavailability assessment of lipidic self-emulsifying formulations of halofantrine. International Journal of Pharmaceutics. 1998;167:155-64. DOI: https://doi.org/10.1016/S0378-5173(98)00054-4

19. Borhade V, Nair H, Hegde D. Design and evaluation of self-microemulsifying drug delivery system (SMEDDS) of tacrolimus. AAPS Pharm Sci Tech. 2008;9:13-21. 2008/05/01. DOI: 10.1208/s12249-007-9014-8.PMC2976874

20. Patil $P$ and Paradkar A. Porous polystyrene beads as carriers for selfemulsifying system containing loratadine. AAPS PharmSciTech 2006; 7: E28-E28. DOI: 10.1208/pt070128

21. Patil P, Paradkar A. Porous polystyrene beads as carriers for self-emulsifying system containing loratadine. AAPS Pharm Sci Tech. 2006;7:E28. 2006/04/06. DOI: 10.1208/pt070128.PMC2750735

\section{PICTORIAL ABSTRACT}

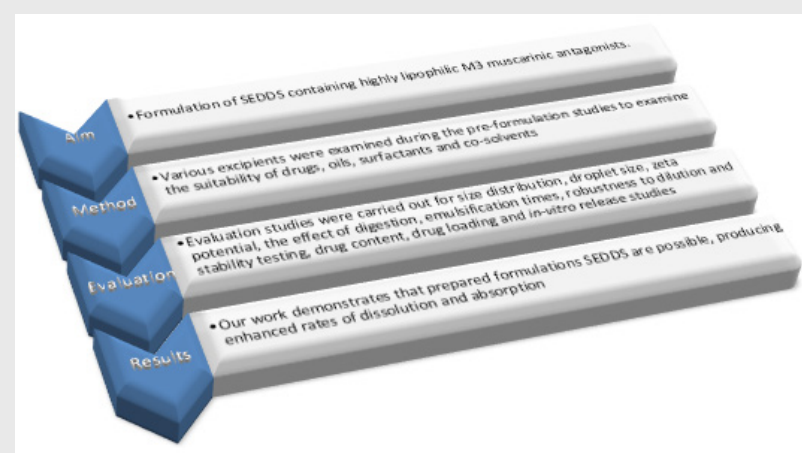

\section{SUMMARY}

- Self-Emulsifying Drug Delivery (SEDDS) system with the poorly hydrophilic drug, darifenacin was developed. The analyses of the ternary phase diagram confirmed that the Smix ratio of 2.1 yeilded optimal results. Additionally, a series of studies were carried out including: visual assessments, thermodynamic stability studies, robustness to dilution and assessment of the efficiency of self-emulsification. Thus, SEDDS may provide a viable alternative for existing formulations of darifenacin on the market.

Cite this article: Harsha NS, Shariff A, Shendkar YA, Al-Dhubiab BE, Meravanige G. Development and Evaluation of a (SEDDS) Self-Emulsifying Drug Delivery System for Darifenacin Hydrobromide. Indian $\mathrm{J}$ of Pharmaceutical Education and Research. 2019;53(2S):s204-s212. 